\title{
INTERNET EN LA EDUCACIÓN INTERCULTURAL: CAMBIO DE ACTITUDES Y VALORES TRAS LA APLICACIÓN DE UN PROGRAMA EN ALUMNADO DE EDUCACIÓN PRIMARIA
}

Resumen: Esta investigación tiene como objetivo evaluar los efectos que el Programa "E-Culturas" ha tenido en alumnos de $6^{\circ}$ curso de Educación Primaria. El programa de contenido intercultural está basado en un enfoque constructivista de aprendizaje y el uso de la red Internet. Para evaluar los efectos del programa se ha utilizado el método cuasiexperimental a través de un diseño pre-postest, por medio del "Cuestionario de Educación Intercultural E-Culturas". La aplicación del programa ha producido efectos satisfactorios en relación a la integración, la convivencia, la tolerancia, el mejor conocimiento cultural y el respeto a otras culturas.

Palabras clave: Internet; nuevas tecnologías de la información y la comunicación; educación intercultural; programa.

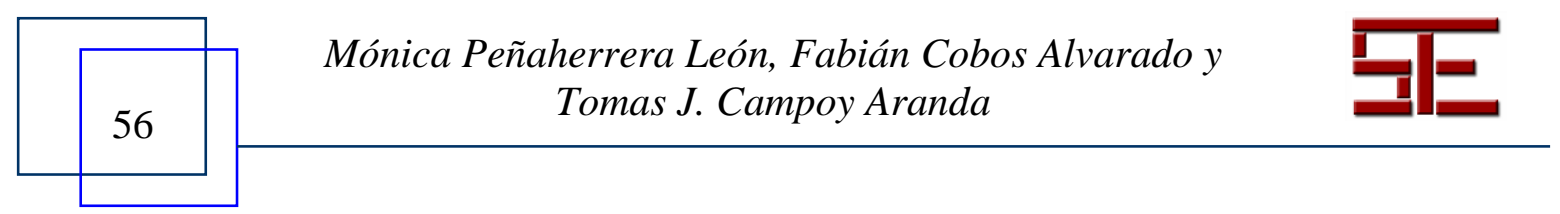




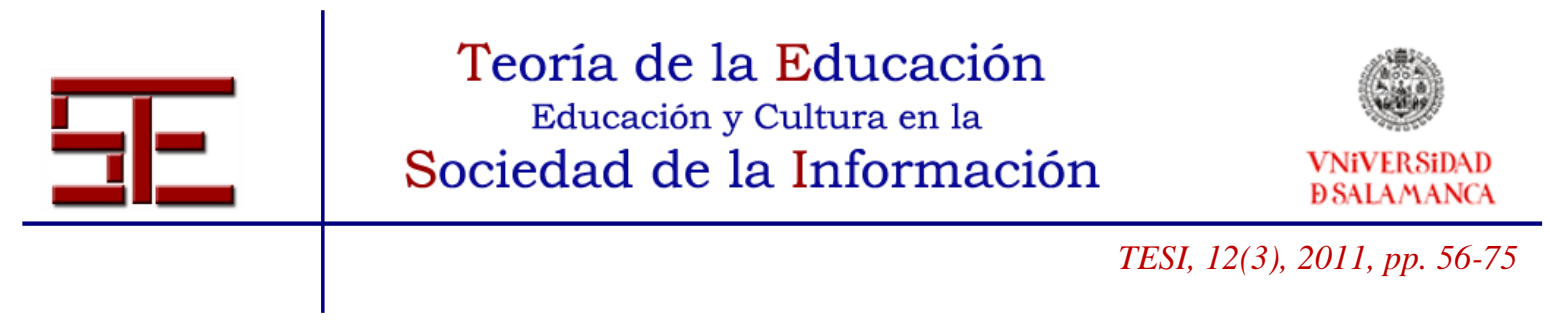

\title{
THE INTERNET IN INTERCULTURAL EDUCATION: CHANGING ATTITUDES AND VALUES AFTER THE IMPLEMENTATION OF A PROGRAM IN ELEMENTARY EDUCATION STUDENTS
}

\begin{abstract}
This research aims to assess the effects of the program "E-Culture" has taken grade 6 pupils in Primary Education. The intercultural program content is based on a constructivist approach to learning and use of the net. To evaluate the effects of the program has used quasi-experimental method through a pre-posttest design, through the "Survey of Intercultural Education E-Culture". The implementation of the program has produced satisfying results in relation to integration, coexistence, tolerance, better cultural understanding and respect for other cultures.
\end{abstract}

Keywords: Internet; new information technologies and communication; intercultural education; program.

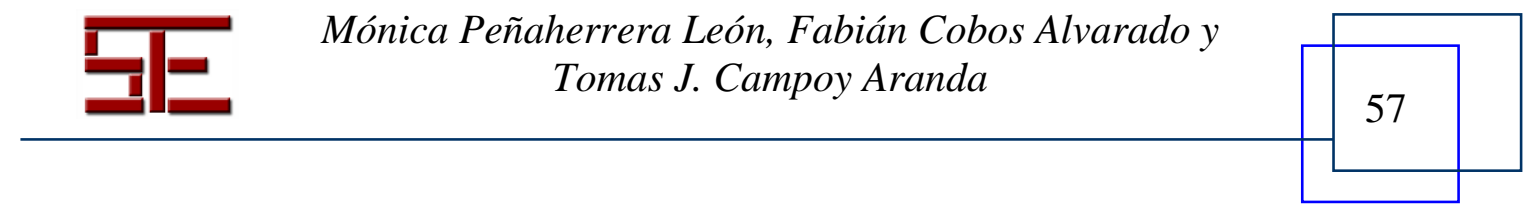




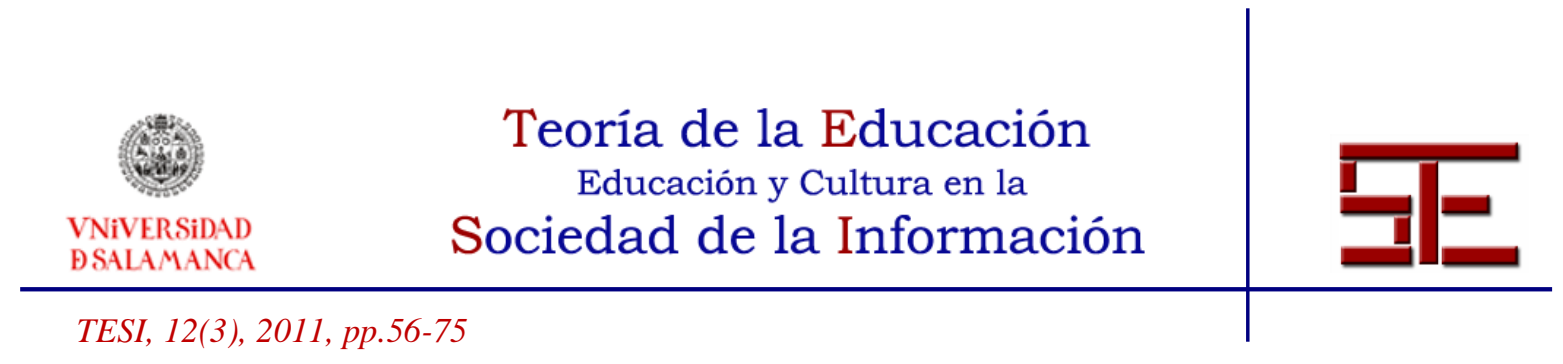

\title{
LA RED INTERNET EN LA EDUCACIÓN INTERCULTURAL: CAMBIO DE ACTITUDES Y VALORES TRAS LA APLICACIÓN DE UN PROGRAMA EN ALUMNADO DE EDUCACIÓN PRIMARIA
}

Fecha de recepción: 21/12/2010; fecha de aceptación: 12/07/2011; fecha de publicación: 30/11/2011

\author{
Mónica Peñaherrera León \\ mpleon@ujaen.es \\ Universidad de Jaén \\ Fabián Cobos Alvarado \\ efabian@gmail.com \\ Universidad de Jaén \\ Tomas J. Campoy Aranda \\ tjcampoy@ujaen.es \\ Universidad de Jaén
}

\section{1.- INTRODUCCIÓN}

En los últimos cincuenta años, el volumen y la importancia de la migración internacional han ido aumentando con rapidez. La globalización trae consigo aumentos de flujos transfronterizos como son los movimientos de personas. El fenómeno multicultural ya es una realidad. Ha transcurrido más de una década de la que hemos pasado de "asombro", "sorpresa, "impavidez" y "expectación" ante la presencia de personas de origen extranjero. Se nos ha hecho "habitual" -no normalidad- la presencia física de inmigrantes en la cotidianeidad española. No obstante, esta presencia está lejos de ser intercultural. Ya que entendemos que la interculturalidad no pasa por el mero contacto con personas de diferentes culturas, va mucho más allá, requiere una meta comprensión del otro, implica comprender el sentido y el significado que tienen para otros tanto su forma de vida como de su ser, considerando que mientras más aspectos significativos se compartan en las relaciones interculturales mayor conocimiento y profundización tendremos del otro.

Aguado, Gil y Mata $(2005,23)$ ponen de manifiesto que para que exista esta profundización es necesario descubrir qué tenemos en común y de esta manera establecer puentes que nos permitan entendernos, y también en qué nos distinguimos de

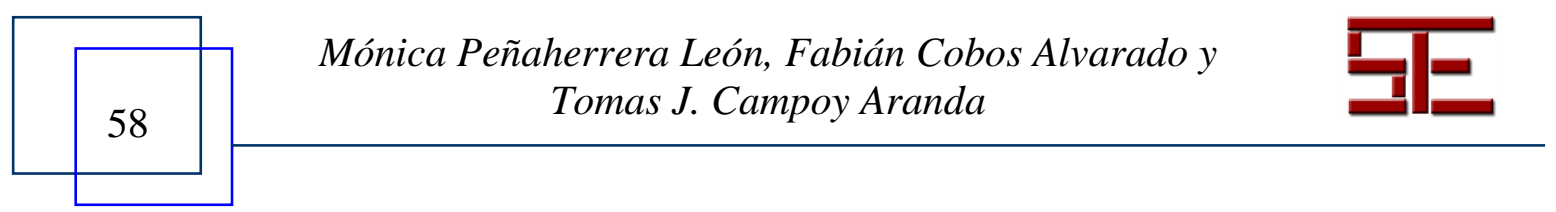




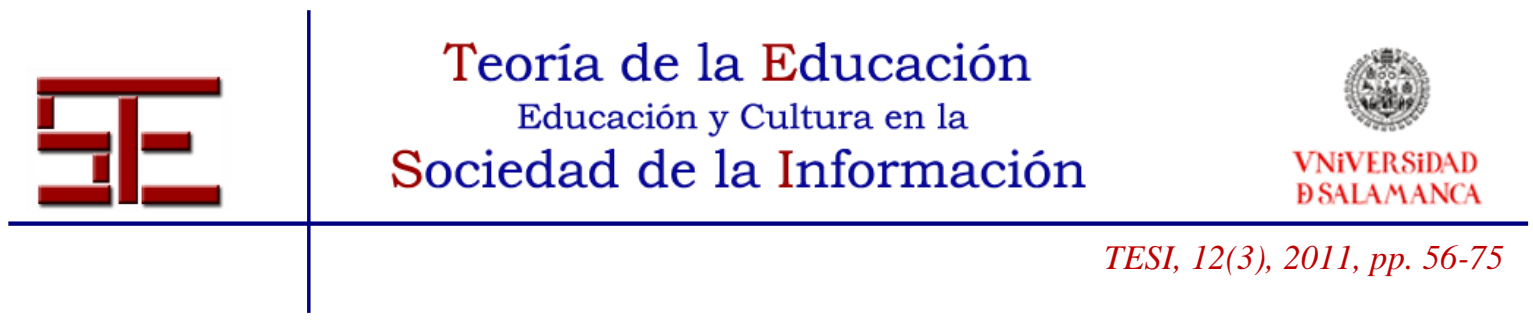

los demás para negociar las expectativas comunes, las normas y valores necesarios para la convivencia.

Por tanto, abordar el fenómeno intercultural en el contexto escolar cada vez es más necesario en la sociedad global en la que vivimos. En palabras de Verde Peleato (2010), nos encontramos ante una situación que nos obliga a replantearnos la estructuración y organización escolar de cara a dar respuestas adecuadas a la diversidad cultural y lingüística.

Pensemos pues, ¿cómo la escuela está propiciando la interculturalidad? ¿Cómo se está gestionando el entramado cultural de aquella diversidad existente en el aula para que exista más "ganancia" que "pérdida".

Parte de las respuestas a estas interrogantes las podemos encontrar en la intervención de Amin Maalouf, premio de las Letras de la Fundación Príncipe de Asturias (2010) cuando expresa que "Lo que importa no es saber si podremos vivir juntos pese a las diferencias de color, de lengua o de creencias; lo que importa es saber cómo vivir juntos, cómo convertir nuestra diversidad en provecho y no en calamidad".

Y ese ¿cómo vivir juntos? en palabras de Llopis y otros (2003), sitúa a la cultura en el nivel profundo, como una herramienta que configura nuestro modo de ser y ayuda a situarnos en el mundo. Entender y vivir la cultura como un fenómeno plural y multiforme supone un proceso global de toda la sociedad y la necesidad de afirmar, a escala mundial, una mentalidad en la que se reconozca el derecho a la diferencia. No olvidemos que la cultura y todo lo que conlleva es un bien común, es una riqueza social y, por lo tanto, debe ser sostenible.

Se trata entonces de hallar claves de comprensión de los problemas del mundo, formar a ciudadanos capaces de interpretar la realidad y participar activamente en su transformación para construir sociedades más incluyentes, más tolerantes, solidarias y comprometidas con el cambio social y mundial.

La propuesta educativa intercultural, por tanto, se basa en conocer y reconocer las culturas sin que una sea superior a la otra; implica entender que las diferencias culturales deben ser valoradas como algo positivo; reconoce los derechos no sólo individuales, sino los que pertenecen a los grupos y colectivos; sostiene el derecho a la diferencia y el enriquecimiento mutuo como uno de los pilares en que se sustenta este tipo de educación (Jordán, 2001).

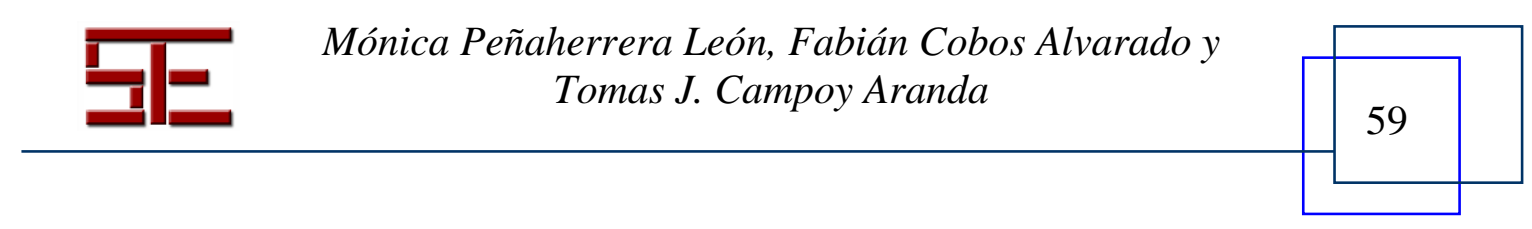




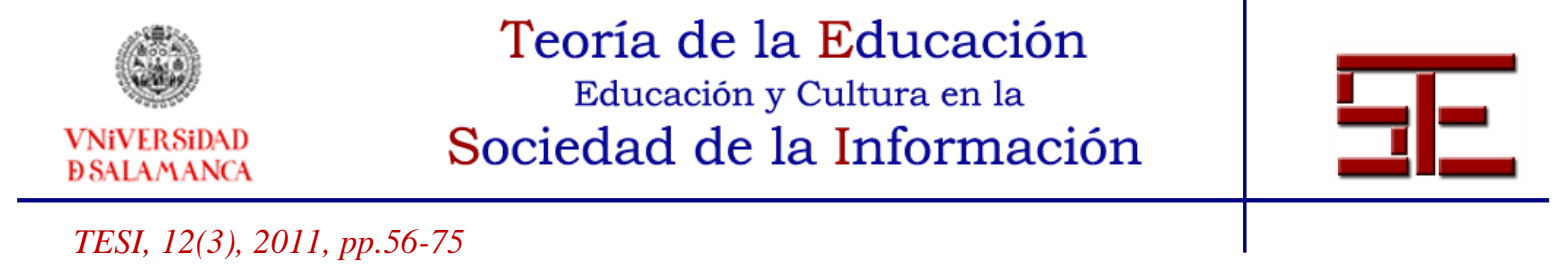

Para Aguado y otros (2005) la educación intercultural propone una práctica educativa en la que debamos interactuar, comunicar, negociar y enriquecernos de grupos culturales diversos, las normas y los valores que el grupo comparte, basados en el reconocimiento de $\mathrm{y}$ en las diferencias individuales/culturales.

Lo anterior se traduce en tener un contacto y acercamiento con otros referentes culturales. Pero este contacto tiene que ser muy particular: tiene que ser de buena calidad, que promueva actividades de cooperación entre los miembros de los grupos, para crear condiciones de igualdad de estatus de manera que cada grupo perciba que está contribuyendo a algo relevante. Además, tiene que darse en un escenario favorable para poder intercambiar experiencias individuales $y$, finalmente, tiene que estar amparado por el profesorado y el contexto escolar en su conjunto.

En opinión de Herring (citado por Malik, 2002) para conseguir un clima intercultural en el centro y en las aulas debemos hacer lo siguiente:

- Agrupamientos flexibles y variados para fomentar la cooperación social.

- Distintas metodologías de enseñanza-aprendizaje para dar respuesta a los diversos estilos de aprendizaje.

- No dar por supuesto que todo el alumnado conoce las normas básicas de funcionamiento de la clase - enseñarlas de forma explícita.

- Interpretar adecuadamente el significado de los comportamientos de los alumnos.

- Hacer énfasis en los aprendizajes significativos: aplicar las estrategias propias de un enfoque constructivista del aprendizaje.

- Conocer las costumbres, creencias y valores de los alumnos de la clase y aprovecharlos como recursos didácticos.

- Trabajar con el alumnado la identificación de estereotipos y de mensajes racistas o sexistas. Elaborar un currículo intercultural en el que se vean identificados de forma positiva todos los grupos presentes en el centro.

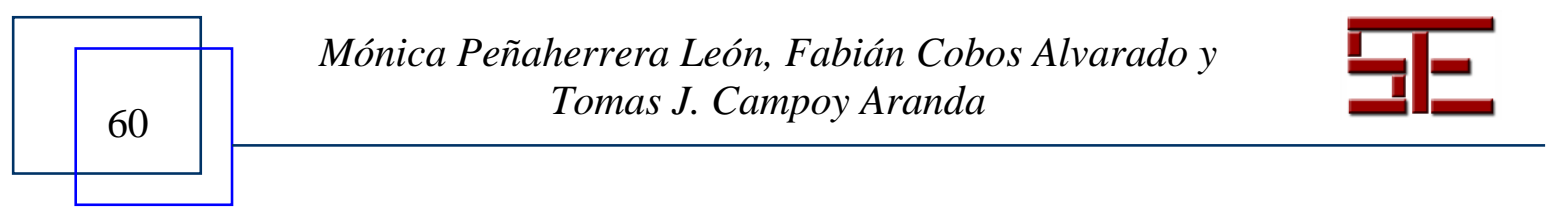




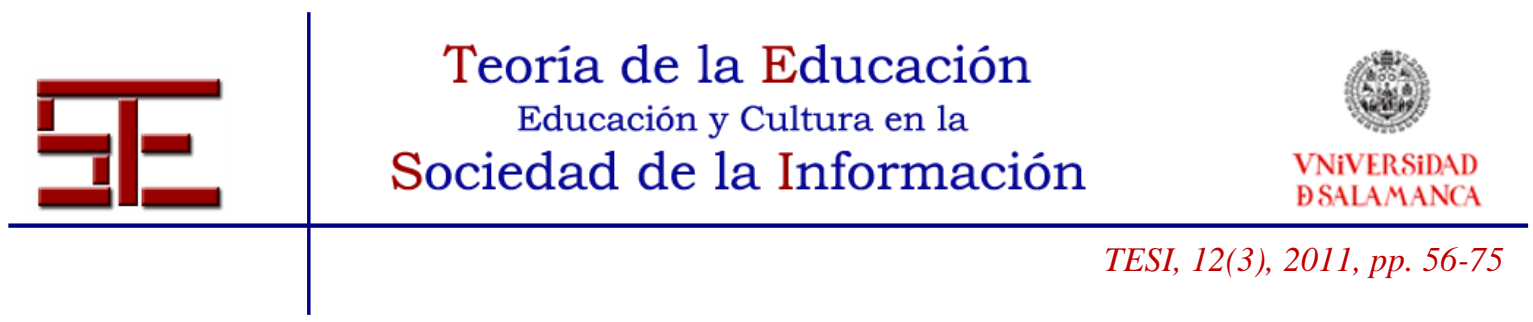

Partimos entonces de la idea de que la interculturalidad se plantea como un constructo en el que se hace necesario un cambio de pensamiento a través de la comprensión de las características distintivas de uno/a mismo/a y de otras culturas (Chen, 1990, citado por Vilá, 2008).

Por otro lado, el avance de las Tecnologías de la Información y la Comunicación (TIC) ha permitido, en los últimos años, cambiar el concepto de "enseñanza" y la forma de obtener conocimiento e información. Cada vez más, con la utilización de distintos medios como el ordenador, Internet, la telefonía móvil, tenemos la posibilidad de diversificar la metodología que se usa en el aula, el intercambio y distintas formas de aprender y conocer. Si nos centramos sólo con Internet y los recursos Web 2.0, el alumno tiene más fuentes de información, más acceso a diversificar sus conocimientos y más contacto con otras realidades culturales y sociales (Peñaherrera y Cobos, 2010, 150).

Así, con el rápido avance tecnológico, los movimientos de población y las nuevas exigencias profesionales, se requiere cada vez más una escuela capaz de desarrollar competencias en el alumnado, capaz de adaptarse a los nuevos escenarios que se van configurando en la sociedad. Corresponde a la escuela el reto de educar en un mismo espacio de convivencia a grupos culturalmente distintos. Esto no excluye, desde nuestro punto de vista, el espacio virtual, en el que todavía es más fácil que coincidan, en que se produzcan relaciones simétricas, que interactúen personas distintas culturalmente, alejadas físicamente, pero muy cercanas telemáticamente.

La cultura comprendida como el conjunto de significados que se construye colectivamente a partir de la interacción, los entornos virtuales y la escuela como escenario más próximo pueden ser las mejores herramientas para potenciar los intercambios, las interacciones y transmitir contenidos culturales para una construcción social de la cultura.

Hasta ahora ha sido muy poca la reflexión sobre el impacto de las TIC en la cultura, pero lo que sí es evidente es que estamos abocados a dos eras: la Sociedad de la Información (SI) y la Sociedad Multicultural y Multiétnica (SMM), que se conjugan de manera simultánea.

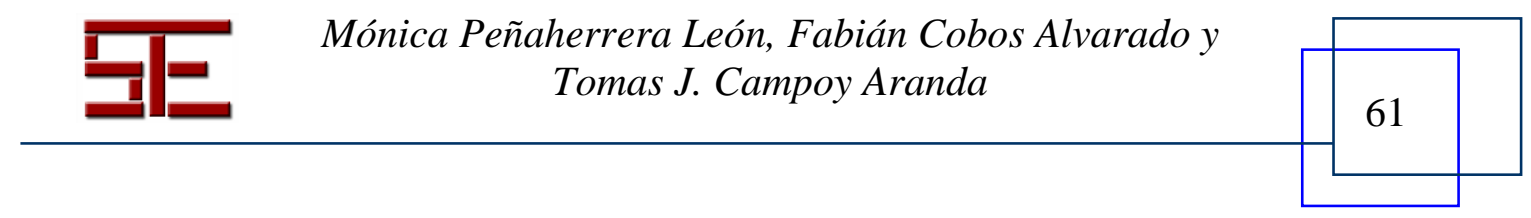




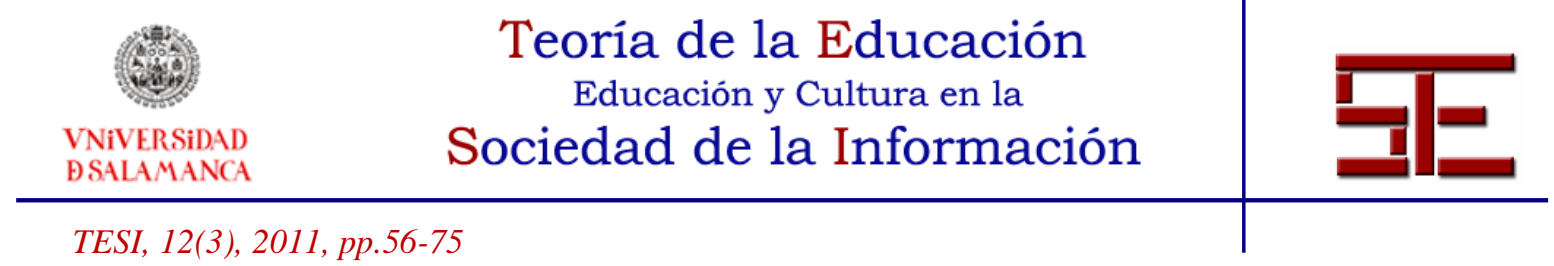

Los avances en la red, por un lado, y las migraciones, por otro, aumentan de manera progresiva. Ya en la red nos encontramos como "nativos" e "inmigrantes" digitales, dos conceptos que tecnológicamente tienen un significado, pero que socialmente también lo están teniendo. De ahí que creemos que se precisa una respuesta adecuada a todos los cambios que se van configurando.

La coincidencia de ambas líneas de actuación (TIC e interculturalidad) en una misma línea propicia la puesta en valor de un postulado: las TIC facilitan el encuentro y la comunicación entre personas distantes a miles de kilómetros y además lo hacen de manera rápida e interactiva.

Justamente esto es lo que se ha abordado a través del programa "E-Culturas", una idea original surgida en un primer momento para apoyar el Plan Andaluz para la inmigración desde una óptica socio - educativa.

Nuestra idea de diseñar, aplicar y evaluar un programa educativo intercultural surge, precisamente, a partir de que son escasos los programas que planteen soluciones prácticas a las actividades cotidianas de los maestros que se enfrentan con el fenómeno multicultural, dotando y nutriendo al programa el uso de las Tecnologías de la Información y la Comunicación (TIC).

Las TIC pueden constituirse en un medio eficaz para dar un tratamiento adecuado a la realidad multiculturalidad. Internet, la videoconferencia (VC), el correo electrónico, y otros de carácter telemático, ofrecen una posibilidad para el contacto y encuentro entre culturas. Estas herramientas tecnológicas favorecerán el acercamiento y disminuirán las distancias culturales que puedan existir entre grupos culturales diferentes, contribuyendo a una sociedad cada vez más intercultural.

En concreto, teniendo en cuenta estas necesidades "nace" el Programa Intercultural ECulturas. Este programa se enmarca en el contexto de la educación intercultural, utilizando como soporte las TIC, con el objetivo de que todo el alumnado tome conciencia y

\footnotetext{
${ }^{1}$ Nativos digitales, los que nacieron con Internet y no la ven como algo tecnológico sino como parte integral de su vida, y los inmigrantes digitales, aquellos que han nacido en la era Antes de Internet (A.I).

2 El programa E-Culturas, es un programa de educación intercultural utilizando TIC. El nombre de este programa, así como su creación y diseño, fue ideado por Mónica Peñaherrera León y Fabián Cobos Alvarado.
}

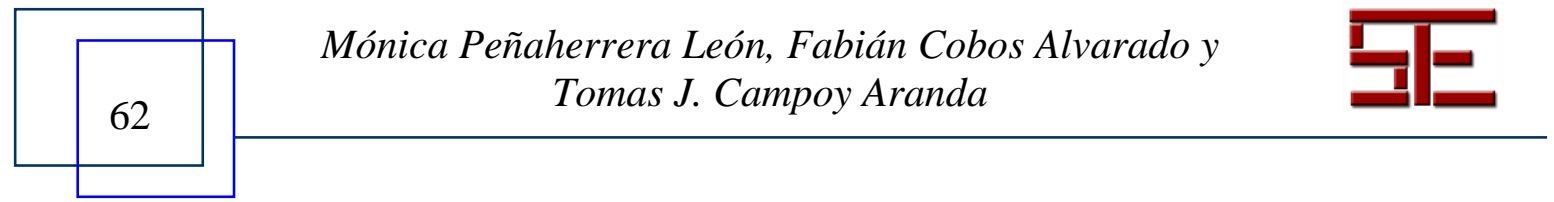




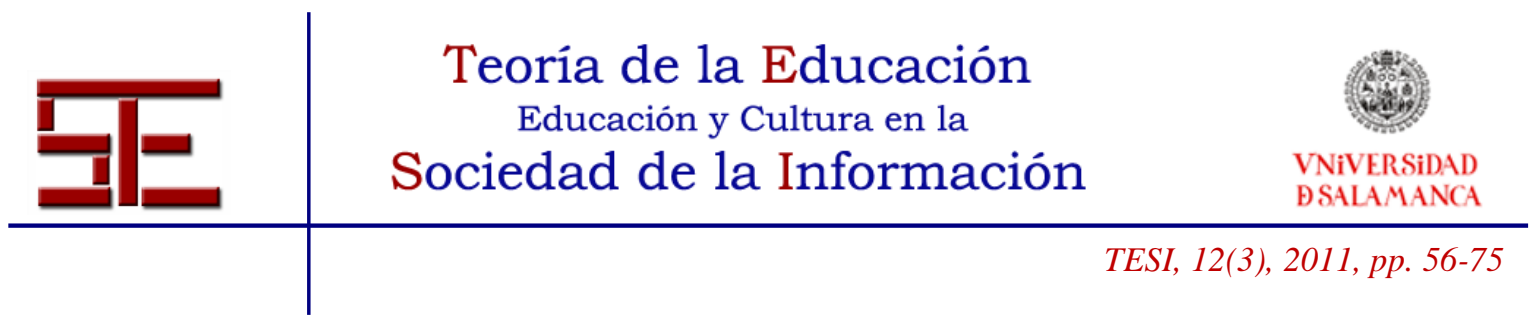

desarrolle actitudes favorables, se fomente la colaboración y el respeto mutuo, a fin de que se traduzca en conductas donde la convivencia marque el estilo de vida.

Este programa ha sido concebido para dirigirse a todos los alumnos, sean o no sean inmigrantes, ya que compartimos el criterio de que la educación intercultural no es un programa exclusivamente para alumnado inmigrante, para determinado grupo que podemos calificar como marginados o desfavorecidos frente a otros. Más bien, se trata de una educación para todos, a fin de que se involucren todos los sujetos en el fenómeno de la inmigración porque en la sociedad en que vivimos estamos "expuestos" siempre a la multiculturalidad.

\section{1.- El programa de educación intercultural E-Culturas}

El Programa "E-Culturas" se caracteriza por la imbricación de la interculturalidad, en el currículo educativo de alumnado de sexto curso de educación primaria.

El Programa se basa en la pedagogía intercultural, basado en un enfoque constructivista del aprendizaje y con apoyo de las nuevas tecnologías de la información y la comunicación, en especial Internet.

El programa ha buscado desarrollar contextos de aprendizaje colaborativo en los que los estudiantes utilizan Internet como herramienta para el desarrollo de las actividades, transferencia de conocimiento, almacenamiento y extracción de la información; de allí que la modalidad de trabajo de este programa sea, sobre todo, colaborativa y con uso de las TIC. Nos referimos también al uso del correo electrónico, chat, foro, Internet, cámara web, ordenadores, etc.

El Programa se ha desarrollado a través de una página web, de acceso libre y con un conjunto de apartados y servicios sobre el programa. Dispone de varias herramientas informáticas.

Contiene una plataforma educativa, que es la zona restringida exclusivamente a usuarios registrados como los administradores, los tutores y los alumnos. Es ahí donde se encuentran los módulos interculturales a los que el alumnado ha tenido acceso con su usuario y pasword.

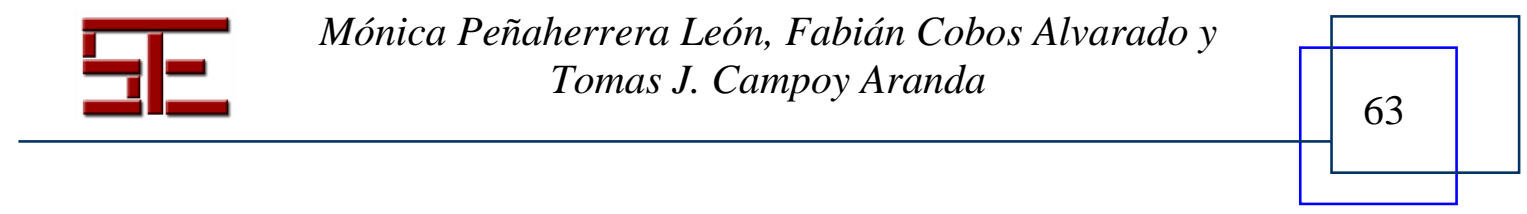




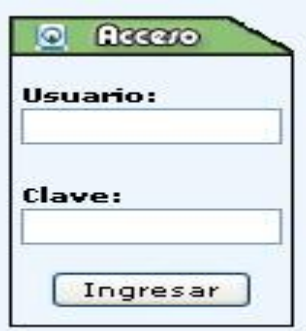

Figura 1. Acceso al área de los módulos.

Dentro de la sección de usuario registrado la plataforma distingue entre tres tipos de usuarios: administrador, profesor-tutor y alumno. Todos tienen un escritorio personal con distintas opciones. El usuario administrador es el que más control tiene sobre el sistema: las diferentes secciones del proyecto, las guías para los profesores, noticias, gestión y foros. A él tienen acceso el webmaster, la investigadora, el tutor de la tesis y otros profesionales que en su momento han formado parte de este proyecto (Figuras 2 y 3 ):

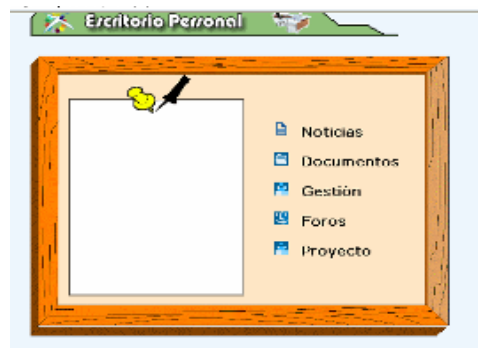

Figura 2. Escritorio del administrador.

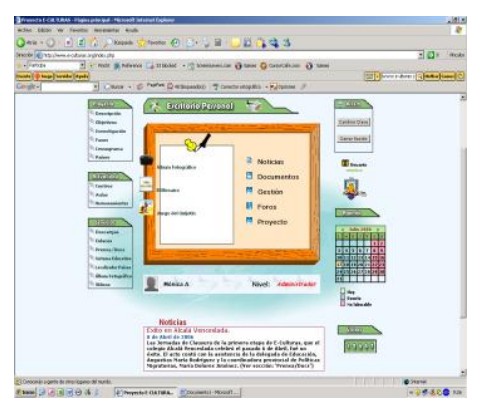

Figura 3. Escritorio del administrador visto desde un ordenador.

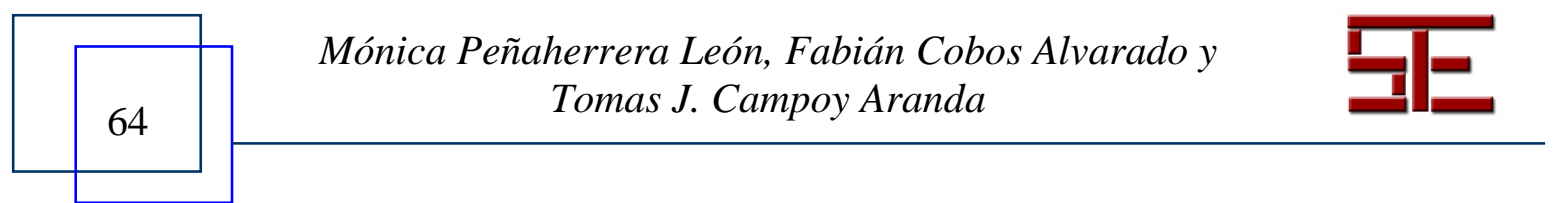




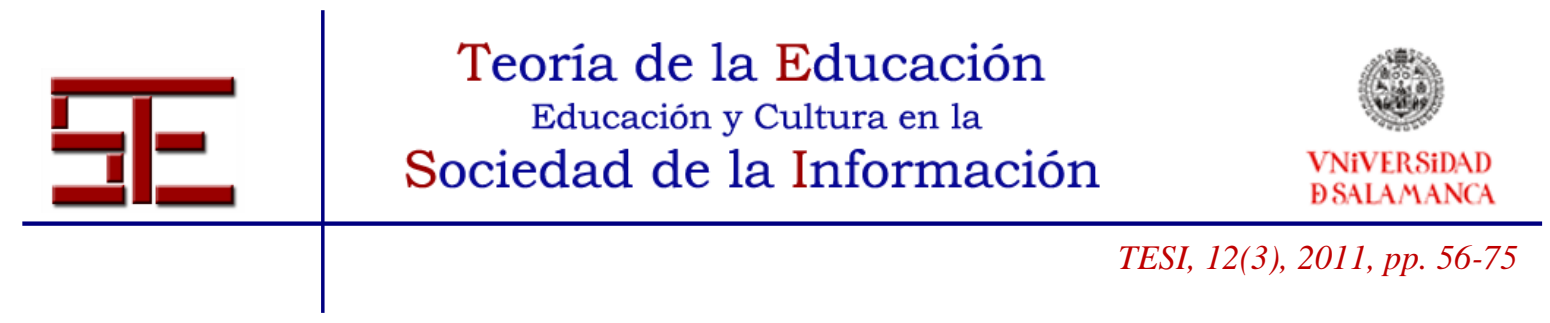

La entrada al programa es personalizada y desde el primer momento el sistema entiende que todos los cambios realizados en los apartados del mismo los ha realizado el alumno que accedió con su clave pesonal, por tanto, se quedan guardados en el sistema al terminar la sesión.

El foro (Figura 4) está estructurado de forma abierta a los siguientes usuarios: tutores y coordinadores (del centro educativo), para los alumnos y para otras personas interesados que quieran accede a él. Con el foro se pretende compartir experiencias, por iniciativa propia o porque dentro de los módulos se han planteado actividades que invitan a los alumnos a participar de éste.

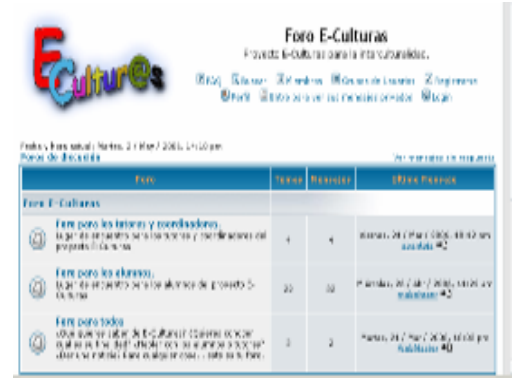

Figura 4. Foro de "E-Culturas".

El programa "E-Culturas" tiene como objetivo general favorecer y potenciar las relaciones interculturales en el alumnado y profesorado español, mediante el diseño y aplicación de un programa educativo que utilice como soporte las TIC para aprender a vivir en sociedades multiculturales.

Los objetivos específicos del programa son los que se anuncian a continuación:

1. Desarrollar estrategias y habilidades cognitivas - análisis, síntesis, representación, elaboración, creatividad- para el desenvolvimiento en una sociedad multicultural.

2. Aplicar módulos interculturales que integren contenidos curriculares.

3. Construir una guía metodológica para cada módulo intercultural.

4. Realizar e intercambiar proyectos pedagógicos que promuevan la participación activa del alumnado participante, utilizando Internet.

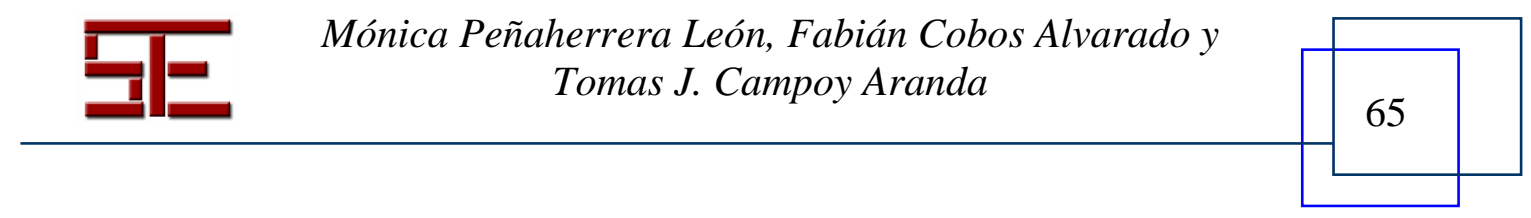




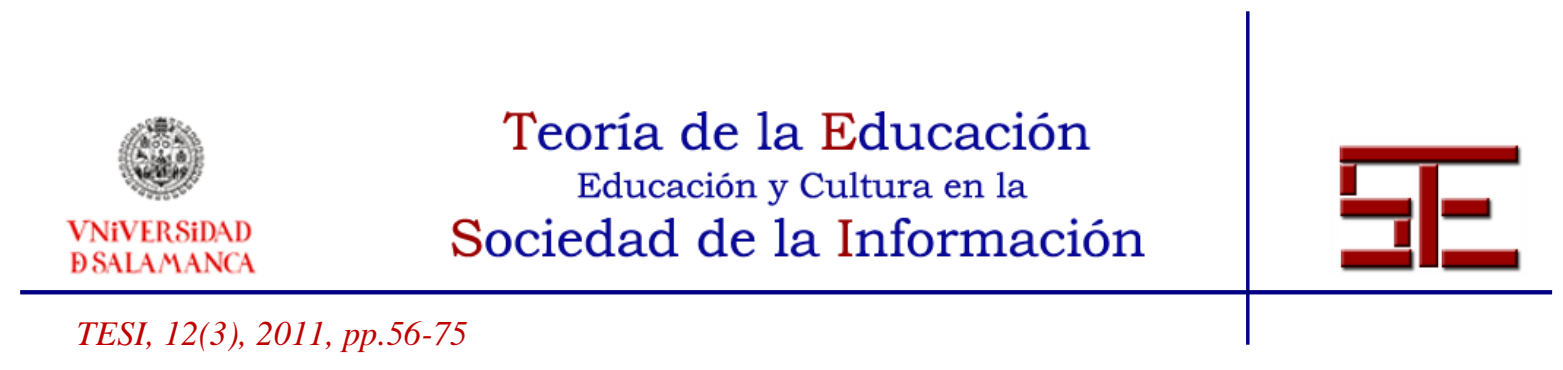

El programa contiene cuatro módulos interculturales con sus respectivas guías didácticas, según aparecen en la Tabla 1. Los módulos que lo componen abarcan las áreas de Conocimiento del Medio y Lengua y tienen un carácter globalizador y transversal. La aplicación de los módulos ha tenido una duración de una hora cuarenta y cinco minutos, 1 día por semana en horas de tutoría.

Tabla 1. Contenidos de los módulos interculturales del programa "E-Culturas".

\begin{tabular}{|c|c|}
\hline Módulo 1 & $\begin{array}{r}\text { El continente americano y el continente europeo: } \\
\text { "Un mundo para todos" }\end{array}$ \\
\hline Módulo 2 & España en Europa, Ecuador en América \\
\hline Módulo 3 & $\begin{array}{c}\text { La diversidad humana: "Hacia una sociedad } \\
\text { intercultural" }\end{array}$ \\
\hline Módulo 4 & España y América antes y después del descubrimiento \\
\hline
\end{tabular}

Para la selección de los contenidos hemos tenido en cuenta:

a. El Real Decreto Decreto 105/92 de 9 de junio de 1992 por el que se establecen las Enseñanzas correspondientes a la Educación Primaria en Andalucía.

b. Los libros de textos utilizados por el alumnado.

\section{2.- NUESTRO ESTUDIO}

\section{1.- Participantes}

El presente estudio se realizó con alumnado de sexto curso del Colegio Público de Educación Infantil y Primaria “Alcalá Venceslada" (Jaén). La edad de estos alumnos gira en torno a 11 años. El número de alumnos que forma del grupo experimental es de 24, de los cuales dos han sido inmigrantes, en concreto, de nacionalidad ecuatoriana.

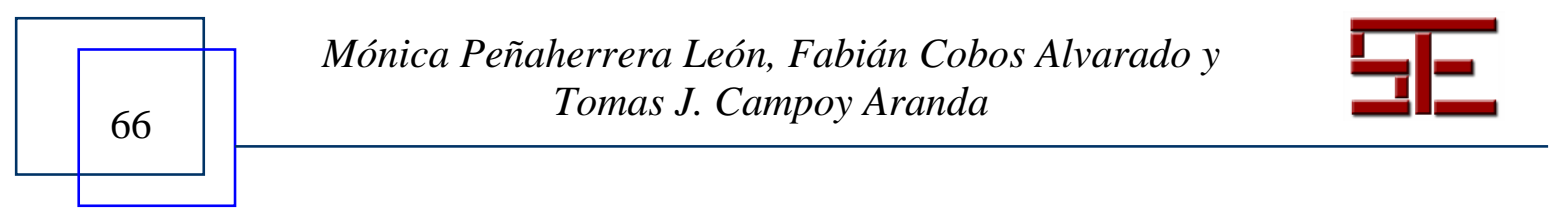




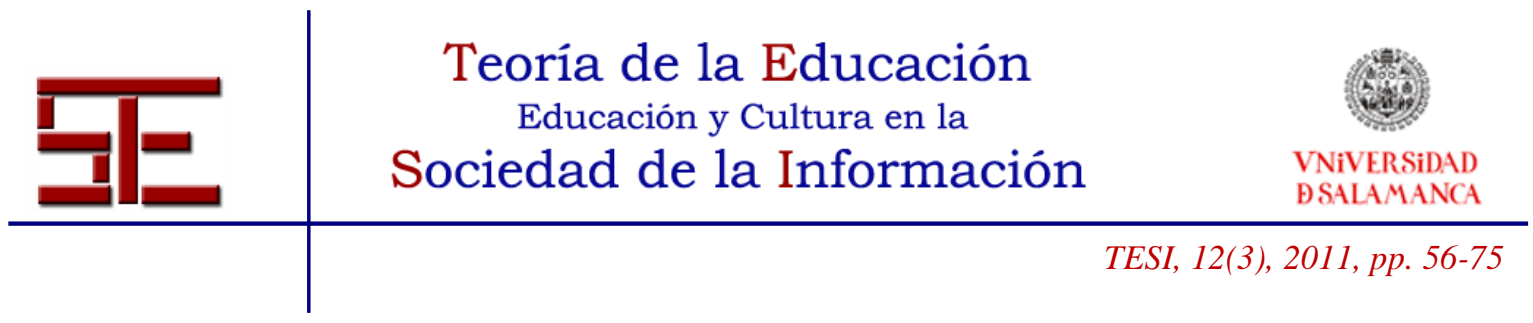

\section{2.- Instrumento}

Para nuestro estudio hemos elaborado ad hoc una escala sumativa, tipo Likert, "Escala de educación intercultural e-Culturas" que, en un momento inicial, estaba compuesta por 78 ítems, tras el estudio piloto se eliminaron aquellos ítems de correlación baja y negativa, quedando en su elaboración definitiva 67 ítems. El número de respuestas al ítem ha sido de cuatro, que ha ido desde "muy de acuerdo" (4) hasta "nada de acuerdo" (1).

Hemos procedido a realizar el análisis factorial y de acuerdo con los indicadores que hemos obtenido (KMO y prueba de esfericidad de Bartlett, etc.), nos indican que la matriz "no es definida positiva". Podemos afirmar que no se dan todas las condiciones necesarias para la aplicación del análisis factorial. En consecuencia, hemos optado por realizar un análisis Clúster, a través de procedimiento de conglomerado jerárquico, al tratarse de nuestro caso de una muestra pequeña. Dicho análisis ha sido realizado mediante el programa estadístico SPSS. v.18., que nos ha permitido establecer las siguientes dimensiones:

1. Conocimiento de sí mismo.

2. Cultura democrática.

3. Justicia social.

4. Sentido positivo de sí mismo.

5. Actitudes hacia la inmigración.

6. Educación ciudadana.

El cuestionario ha sido validado también a través del programa estadístico SPSS. v.18, obteniendo un coeficiente de fiabilidad (alfa de Cronbach) de .70.

\section{3.- Procedimiento}

El procedimiento que hemos seguido se concreta en las siguientes etapas:

$1^{a}$ Etapa: en esta fase recogimos información para el diseño del programa. Posteriormente se elaboró y aplicó el instrumento de evaluación. Se realizó la presentación del Programa a la Administración Pública y al profesorado del Centro. Al mismo tiempo se solicitaron los permisos pertinentes.

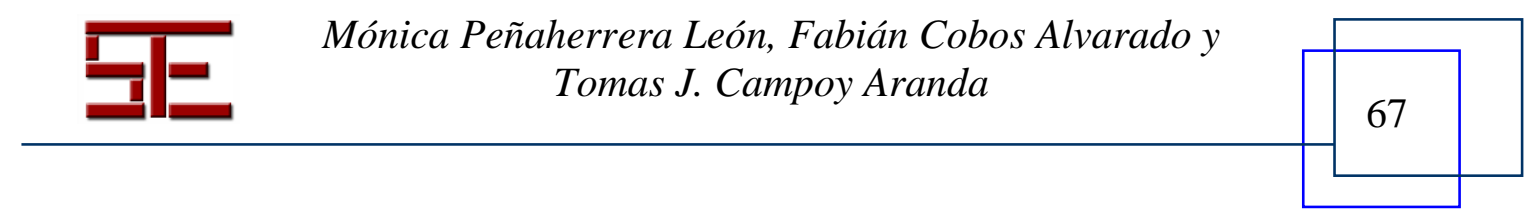




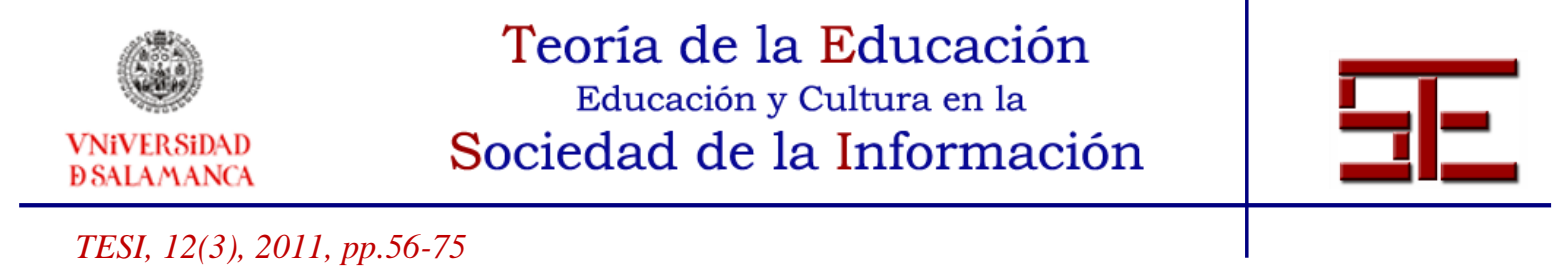

$2^{\text {a }}$ Etapa: se realizó la capacitación del profesorado y la implementación del programa. Se llevó a cabo un seguimiento y registro de todas las incidencias.

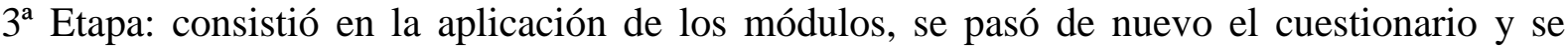
procedió a la recogida e interpretación de los datos obtenidos. Se terminó con la elaboración de conclusiones y propuestas.

\section{4.- Diseño de la investigación}

Con la finalidad de medir los "efectos" del Programa E-Culturas en los destinatarios, hemos aplicado el método cuasiexperimental y, en concreto, el diseño de grupo único, pretest-postest "de grupo no equivalentes", mediante el cual hemos pretendido analizar relaciones de causalidad mediante la manipulación de la variable independiente, partiendo de un grupo ya formado de manera natural. Para ello hemos utilizado un grupo de alumnos de $6^{\circ}$ de Educación Primaria (grupo experimental), al que se le aplicó el pretest y, posteriormente, el postest una vez finalizada la aplicación del programa.

Tabla 2. Diseño de investigación cuasi-experimental

\begin{tabular}{|c|c|c|c|c|c|}
\hline Grupo & Tamaño & Asignación & Pretest & Tratamiento & Postest \\
\hline 1 & $\mathrm{~N}$ & No azar & $\mathrm{X}_{1}$ & $\mathrm{~T}_{\mathrm{o}}$ & $\mathrm{X}_{2}$ \\
\hline
\end{tabular}

\section{5.- Análisis de datos}

Con las respuestas dadas al cuestionario hemos procedido a analizar los indicadores para determinar si procede realizar el análisis factorial. De los distintos indicadores no todos han mostrado la conveniencia (coeficientes, niveles de significación, determinante, KMO y prueba de esfericidad de Bartlett, etc.) de llevar a cabo dicho análisis, por lo que hemos procedido a un análisis clúster (mediante el programa estadístico SPSS v.18). Dado el número reducido de variables optamos por el tipo de análisis de "conglomerados jerárquicos", cuyo procedimiento consiste en identificar grupos homogéneos de casos (variables) basándose en un algoritmo, comenzando por cada variable en un conglomerado distinto y asociando los conglomerados hasta que sólo queda uno. En este caso, hemos tenido en cuenta las elecciones de las variables

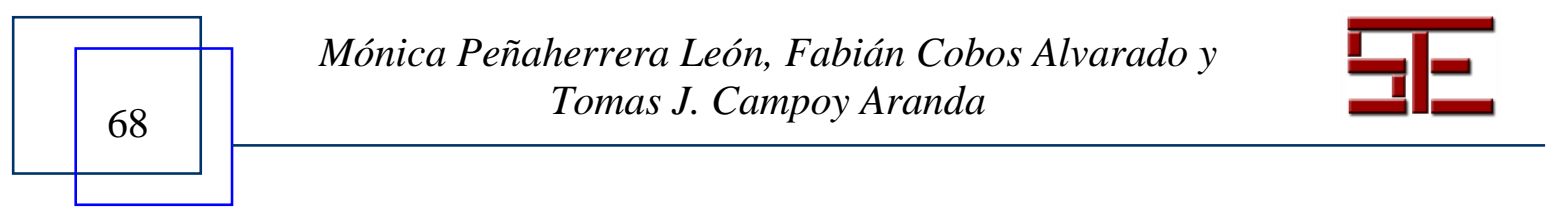




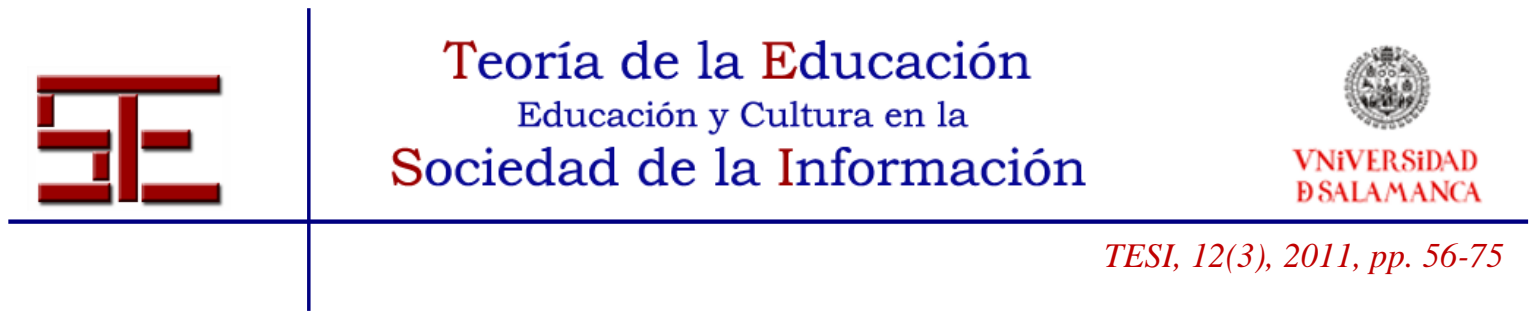

para el análisis, las medidas de proximidad, los criterios para agrupar en conglomerados y la elección del número de conglomerados.

De acuerdo con esto, los ítems han quedado agrupados en las siguientes dimensiones:

1. Conocimiento de sí mismo.

2. Cultura democrática.

3. Justicia social.

4. Sentido positivo de sí mismo.

5. Actitudes hacia la inmigración.

6. Educación ciudadana.

\section{Resultados}

Para el contraste de las hipótesis formuladas en la investigación, hemos utilizado la prueba t de Student. Esta prueba nos ha ayudado a conocer los efectos del Programa "ECulturas" una vez aplicado el mismo al alumnado.

De lo que se trata es de demostrar si las medias entre el pretest y el postest, una vez aplicado el programa, se deben en cada caso a las variables que las diferencia o, si por el contrario, las diferencias son debidas al azar. Es decir, el procedimiento prueba t para muestras relacionadas compara las medias de dos variables de un solo grupo, calcula las diferencias entre los valores de las dos variables de cada caso y contrasta si la media difiere de 0 .

Del total de 67 hipótesis podemos confirmar que resultan significativas 43, lo que supone el $64,2 \%$.

A continuación ofrecemos una síntesis de los principales resultados obtenidos en nuestra investigación, de acuerdo a las dimensiones resultantes del análisis Clúster realizado:

1. Conocimiento de sí mismo

Se han producido cambios positivos en las variables "Confío en mis compañeros", "Me gusta tener amigos inmigrantes", "Cuando hay un problema trato de ayudar a

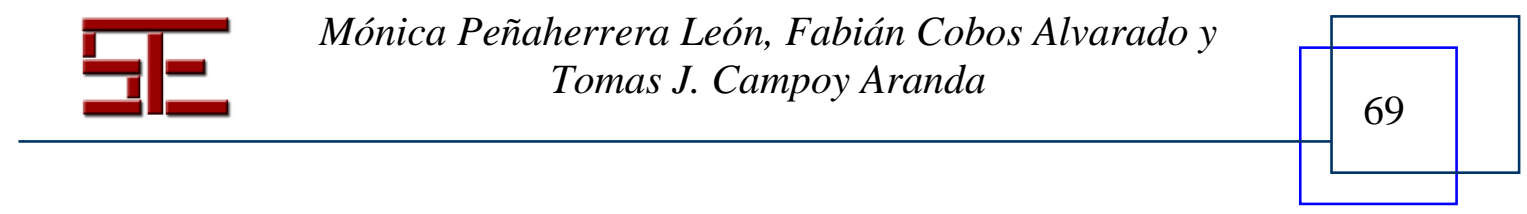




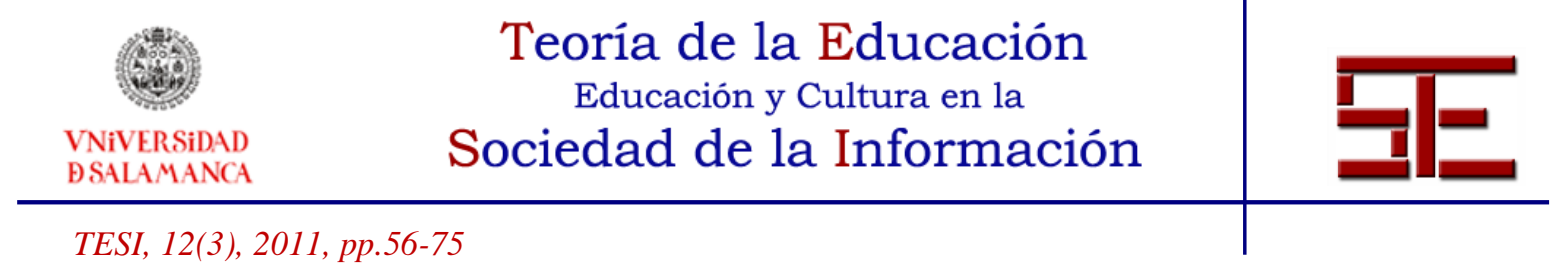

resolverlo", "Tengo buena opinión de los inmigrantes" y "Ayudo a mis compañeros inmigrantes".

Por el contrario, el programa no ha producido mejoras en las variables "Conozco por qué las personas inmigrantes abandonan sus casas para ir a otro país", "Mis profesores piensan que soy buen estudiante", "Hago bien los deberes escolares", "Tengo buenas cualidades" y "Soy cariñoso con los demás".

Por lo tanto, podemos afirmar que el programa en su dimensión "Conocimiento de sí mismo", la mitad de las variables han producido efectos favorables en relación al grupo experiemental.

Consideramos positivo que se haya producido una mejoría en el alumnado en cuanto a la opinión respecto a los inmigrantes y una actitud de solidaridad hacia ellos.

\section{Cultura democrática}

Se han producido cambios positivos en las variables "Acepto a los compañeros que son inmigrantes", "Estoy satisfecho con lo que tengo", "Me llevo bien con mis compañeros", "Me gusta ayudar a mis compañeros".

Por el contrario, el programa no ha producido mejora en las variables "Todos los niños tienen el derecho de satisfacer sus principales necesidades", "Soy una persona feliz", "Creo que me entiendo a mí mismo", "Respeto la forma de ser de mis compañeros inmigrantes", "Resulta difícil que los inmigrantes entren a España".

Como valoración de esta dimensión se puede afirmar que el programa ha producido cambios positivos en el alumnado autóctono, en relación a una actitud de apertura y aceptación respecto al alumnado inmigrante. Sin embargo, el alumnado a esta edad no tiene conocimiento acerca de las normas por las que se regula la entrada de los inmigrantes a España.

\section{Justicia social}

Se han producido cambios positivos en las variables "Me gusta participar en las actividades del colegio", "Cuando molesto a alguien procuro pedir perdón", "Suelo caer bien a los demás", "Separo a mis compañeros cuando discuten", "Todos somos iguales independientemente del color de la piel", " Todas las personas tienen los mismos

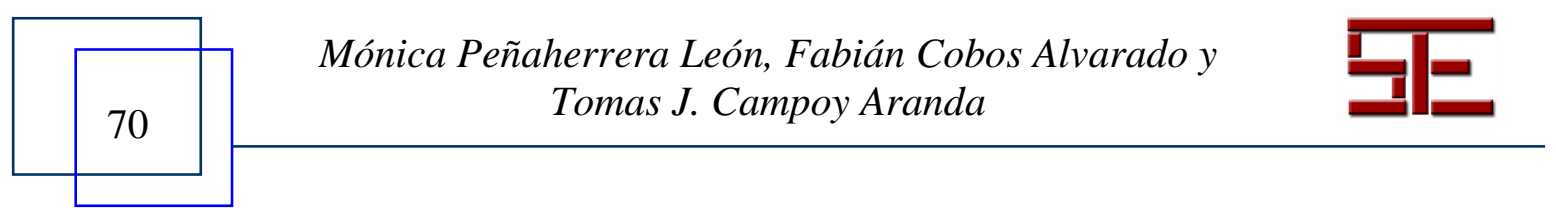




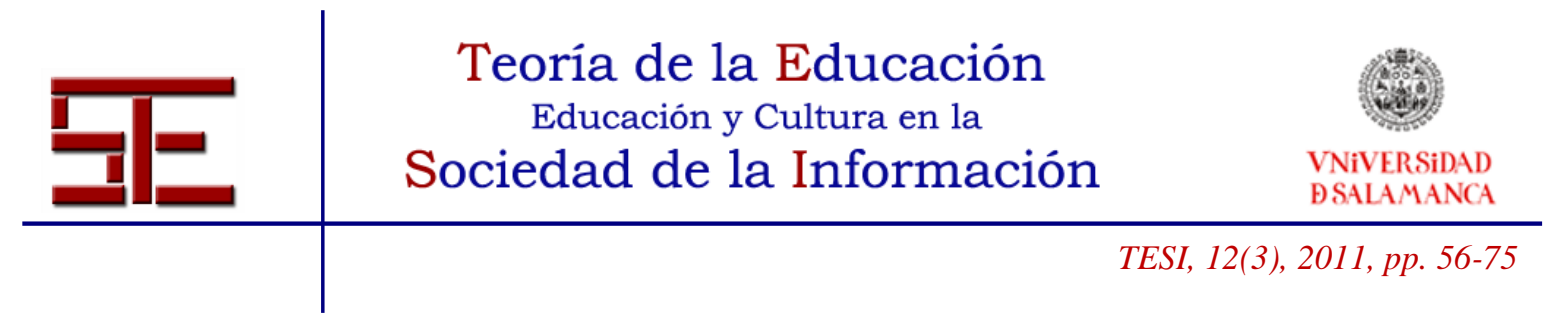

derechos y deberes", "Me encuentro muy a gusto en el colegio", "Los inmigrantes realizan el trabajo que los españoles no quieren", "Pienso que la naturaleza no se respeta lo suficiente", "Los inmigrantes vienen de países más pobres que el nuestro".

Por el contrario, el programa no ha producido mejora en las variables "En mi colegio estudiamos otras culturas diferentes a la mía", "Mi familia me considera algo importante", "Si mis amigos están preocupados yo también estoy preocupado", "Tengo buenas ideas", "Me gusta decidir por medio del voto".

Los resultados de la aplicación del programa ponen de manifiesto que ha habido una mejora en el alumnado con relación "al marco de convivencia". El programa ha producido efectos positivos en cuanto al conocimiento de la situacion social de los inmigrantes en España.

Se ha producido una mejora en la toma de conciencia con relación a la igualdad de todas las personas, independiente de su situación o condición.

Asimismo, se pone de relieve que la programación del centro educativo no contempla suficientes contenidos y actividades relacionados con la educación intercultural.

4. Sentido positivo de sí mismo

Se han producido cambios positivos en las variables "Me considero una persona aceptada", "Me cuesta trabajo hablar de mis sentimientos con mis amigos", "Estoy satisfecho con lo que hago", "Hay cosas de mi manera de ser que no me gustan", "Cuando pienso de manera diferente a los demás me callo", "Los inmigrantes son mano de obra barata", "Me cae bien toda persona que conozco", "Me resulta difícil juntarme en mi colegio con mis compañeros inmigrantes".

Por el contrario, el programa no ha producido mejora en las variables "Sé de qué países vienen los inmigrantes", "Me desanimo cuando algo me sale mal", "Creo que dependo demasiado de los demás".

En esta dimensión, consideramos que un aumento de la mejora del sentido positivo de uno mismo tiene consecuencias importantes en las relaciones con los demás, más dinámicos y reconocimiento de la identidad cultural de otros.

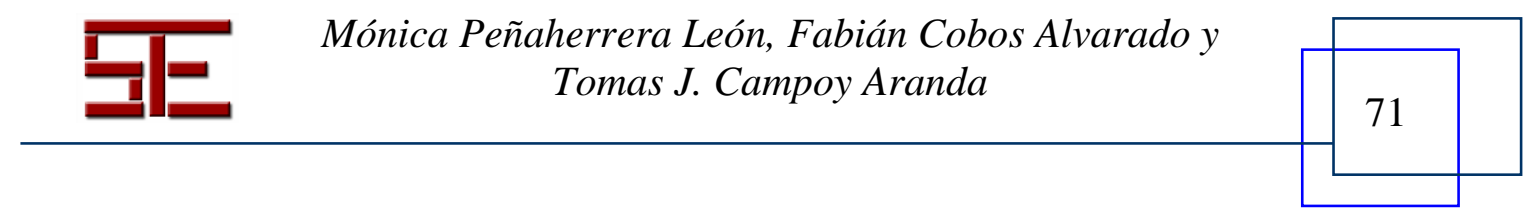




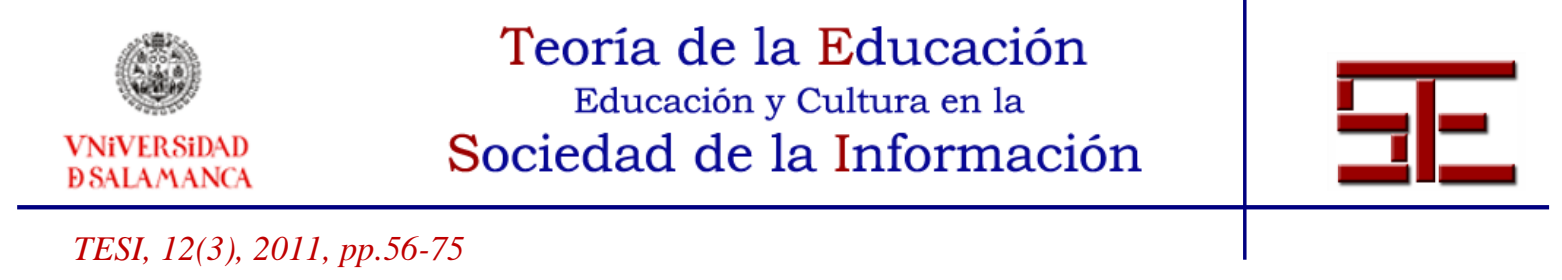

5. Actitudes hacia la inmigración

Se han producido cambios positivos en las variables "Me siento incómodo cuando trabajo en grupo", "Los inmigrantes son personas peligrosas", "Me enfado si los demás no hacen lo que yo digo", "Me parecen graciosos los comentarios racistas", "Me molesta cumplir las normas de clase", "Prefiero trabajar solo antes que hacerlo en grupo".

Por el contrario, el programa no ha producido mejora en las variables "Me gustan las peleas y las riñas", "Se puede discriminar a las personas por razón de sexo, religión, etc", "Tengo manía a las personas que no son como yo", "Soy más inteligente que las personas de otras culturas", "Cada uno debe resolver sus propios problemas".

En el alumnado participante del programa ha habido un cambio sustancial en el sentido de una mayor pertenencia al grupo, una mejor actitud para trabajar en actividades de grupo y aceptación de las normas de grupo. Asimismo queda de manifiesto que se ha mejorado actitudes positivas hacia la inmigración en cuanto al rechazo de frases esterotipadas (me parecen graciosos los comentarios racistas) o superación de prejuicios (los inmigrantes son personas peligrosas).

6. Educación ciudadana

Se han producido cambios favorables en las variables "Pierdo mi paciencia fácilmente", "Conozco cómo viven los inmigrantes en España", "Conozco las culturas hispanas (ecuatorianas, colombianas, etc.)", "Digo la verdad aunque me perjudique", "Conozco cómo se vive en los países de las personas que emigran", "Creo que mi cultura es la mejor".

Por el contrario, el programa no ha producido mejora en las variables "Suelo hacer siempre lo que quiero", "Me desagrada aceptar los acuerdos por mayoría", "Conozco los trabajos que los inmigrantes realizan en España".

El programa ha producido efecto en cuanto al conocimiento que el alumnado autóctono tiene en relación a sus compañeros inmigrantes. El programa ha despertado interés por el conocimiento de la situación de los países de origen de los compañeros inmigrantes, así como en los aspectos sociales y culturales de esos países.

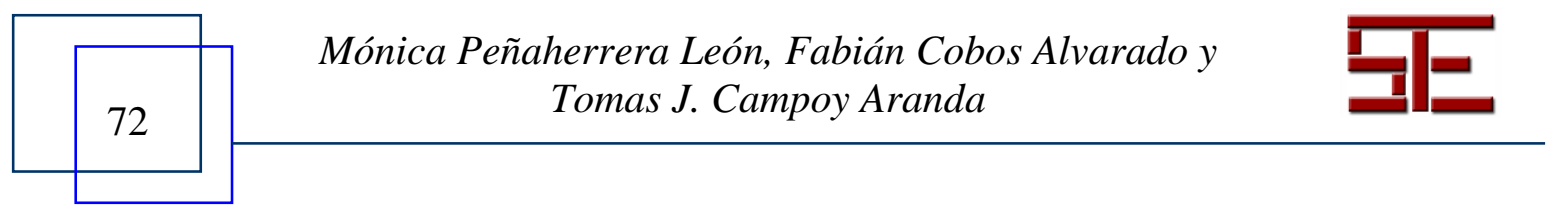




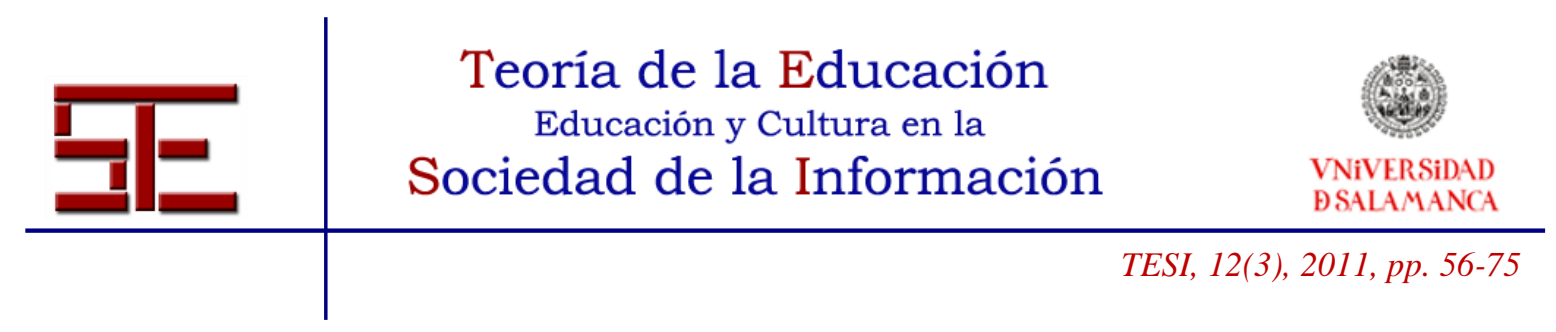

\section{CONCLUSIONES Y DISCUSIÓN}

Algunos autores recomiendan hacer una generalización analítica, es decir, la ampliación de los planteamientos teóricos existentes en función de la evidencia obtenida (Pino Justo y Soto Carballo, 2010). Dicha consideración la hemos tenido muy en cuenta para nuestras conclusiones.

En nuestro estudio podemos concluir que ha habido un cambio significativo en las actitudes y los valores del alumnado con respecto al fenómeno intercultural. El internet y los distintos medios telemáticos han contribuido a experiencias interculturales más significativas entre los miembros del grupo multicultural.

Creemos, que el aprendizaje intercultural es más significativo cuando se explora, experimenta e investiga, y esto es justamente lo que el alumnado ha realizado a través de los distintos contenidos interculturales utilizando Internet. Hemos propiciado que el alumnado por sí mismo busque la información, analice, confronte, compare y cree su "propia teoría", se despoje de ciertos esquemas de partida con repecto a a la inmigración, como, por ejemplo, "vienen porque son pobres", o ciertos prejuicios con respecto a la forma de vida de otros paises "en América sólo hay reguetón y culebrones”. Creemos que los contenidos de los módulos interculturales y su aplicación, utilizando como soporte las tecnologías, han favorecido el encuentro, la integración y la mejora en el grado de relación intercultural de los alumnos.

Es preciso que las experiencias que permitan adquirir aprendizaje intercultural impregnen el currículo de forma transversal, a lo largo de todas las áreas y niveles" (Aguado, 2003, 150). No basta con enseñarles a los niños que existen otros grupos culturales, sino que es necesario que interactúen y construyan experiencias, aprendizajes y proyectos comunes con dichos grupos. De ahí que los contenidos interculturales de nuestro programa han tenido un carácter transversal en los contenidos curriculares.

Hemos de señalar también que, para favorecer la integración, la indentidad y el reconocimiento a la pluralidad cultural, se requiere, a nuestro juicio, una mayor capacidad creativa para favorecer los encuentros y que estos no sean esporádicos ni débiles, sino muy por el contrario, permanentes y de calidad. Poner en práctica nuevas ideas y formas de adaptación y organización curricular, el espacio de aula, los tiempos, etc., exige también un trabajo comprometido y colaborativo del equipo docente del centro escolar. Y, sobre todo exige cambios hacia una cultura que aprende (tutores, mejora de la metodología didáctica, proyectos conjuntos, trabajo con padres, etc.).

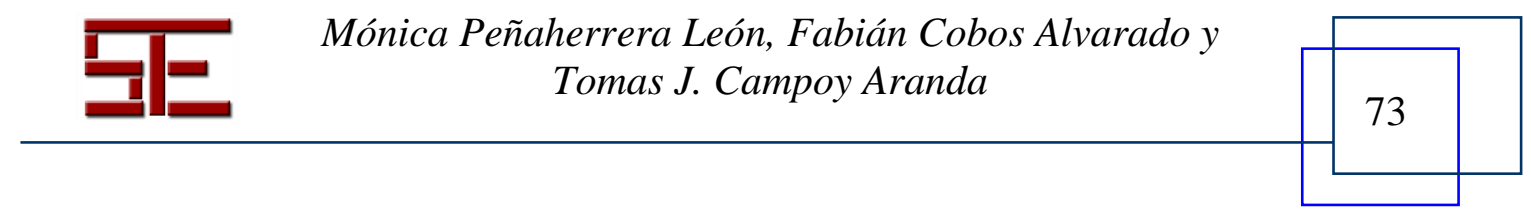




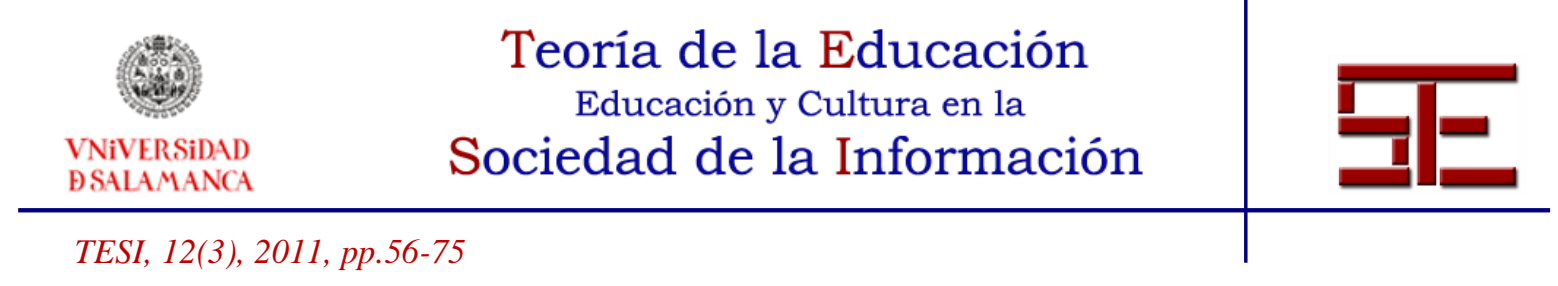

Creemos también que el profesorado debería recibir formación específica en diseño y elaboración de materiales interculturales, conocimiento pedagógico e intercultural de medios tecnológicos, así como el funcionamiento de una plataforma con contenido intercultural.

El programa ha despertado mucho interés en sus destinatarios por su carácter interactivo, los propios contenidos y actividades y la forma de abordar el aprendizaje tanto a nivel metodológico como los recursos tecnológicos empleados. Tanto el profesorado del centro como los padres y madres del alumnado han manifestado su satisfacción por la aplicación de dicho programa.

Para concluir este apartado, creemos que un programa con estas características debería aplicarse a otros niveles educativos, como el de secundaria.

\section{BIBLIOGRAFÍA}

Aguado, T. (2003). Pedagogía Intercultural. Madrid: Mc Graw Hill.

Aguado, T., Gil, I. y Mata, P. (2005). Educación intercultural una propuesta para la transformación de la escuela. Madrid: MEC.

Jordán, J. A. (Coord.) (2001). La educación intercultural, una respuesta a tiempo. Barcelona: EDIUOC.

Llopis, C. y otros. (2003). Recursos para una educación global. ¿Es posible otro mundo? Madrid: Narcea.

Malik Liévano, Beatriz (2002). Modelos de Orientación e Intervención Psicopedagógica. Proyecto Docente. Madrid: UNED. Inédito.

Peñaherrera, M. (2004). Propuesta organizativa para atender la interculturalidad en los centros educativos. En E. Soriano (Coord.), Recursos para la enseñanza y aprendizaje de la Educación Intercultural. (pp. 49-53). Almería: Diputación Provincial de Almería.

Peñaherrera, M. y Cobos, F. (2010). De la exclusión a la inclusión: Un programa para la convivencia, ciudadanía e inclusión en las aulas españolas mediante el uso de la Web 2.0.

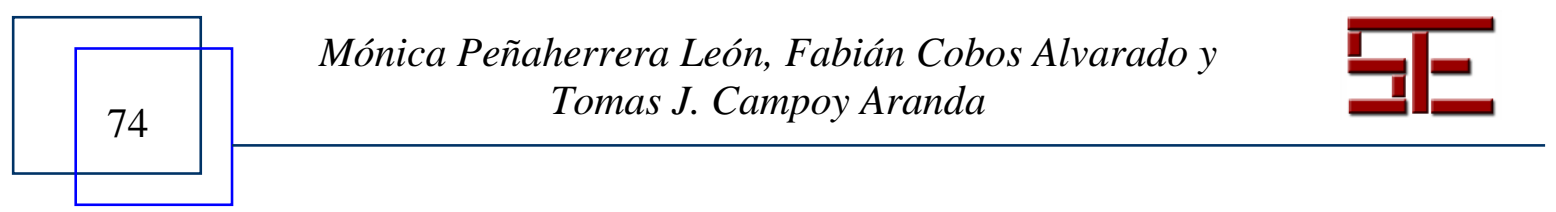




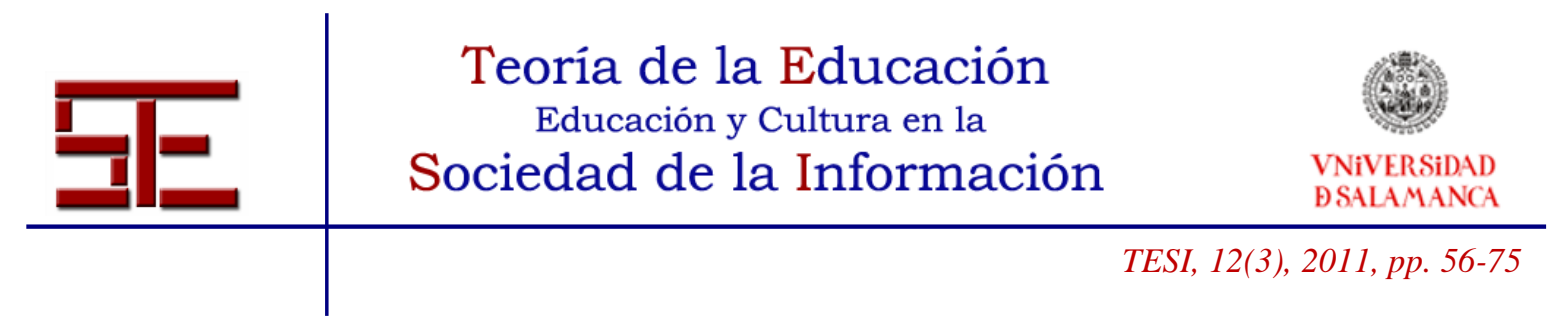

En Ruiz, J. y Sánchez, J. (Coord), Investigaciones en buenas prácticas con Tecnologías de la Información y la Comunicación (pp. 149-158). Málaga: Aljibe.

Pino Juste, M. y Soto Carballo, J. (2010). Identificación del dominio de competencias digitales en el alumnado del grado de magisterio. En Orejudo González, J.P. (Coord.) Perspectiva educativa y cultural de "juego de rol". Revista Teoría de la Educación: Educación y Cultura en la Sociedad de la Información. vol. 11, n 3, 336-362 [Fecha de consulta: 13/noviembre/2010].

http://campus.usal.es/ revistas_trabajo/index.php/revistatesi/article/view/7466/7483.

Premios Príncipe de Asturias de las Letras (2010). Intervención de Amin Maalouf [Fecha de consulta: 13/noviembre/2010].

http://www.clubmadrid.org/img/secciones/Discurso_Maalouf_premios_principe_Asturias_ 2010.pdf .

Vilá, R. (2008). La competencia comunicativa intercultural en adolescentes. Infancia y Aprendizaje, 31 (2), 147-164.

\footnotetext{
NOTAS

${ }^{1}$ Nativos digitales, los que nacieron con Internet y no la ven como algo tecnológico sino como parte integral de su vida, y de los inmigrantes digitales, aquellos que han nacido en la era Antes de Internet (A.I).

${ }^{2}$ El programa E-Culturas es un programa de educación intercultural utilizando TIC. El nombre de este programa, así como su creación y diseño, fue ideado por Mónica Peñaherrera León y Fabián Cobos Alvarado. En el 2006, obtuvo un reconocimiento de la Junta de Andalucía, a través de Políticas Migratorias, en la VI edición de los Premios "Andalucía de las Migraciones".
}

Para citar el presente artículo puede utilizar la siguiente referencia:

Peñaherrera León, M., Cobos Alvarado, F. y Campoy Aranda, T. J. (2011). Internet en la educación intercultural: cambio de actitudes y valores tras la aplicación de un programa en alumnado de educación primaria. Revista Teoría de la Educación: Educación y Cultura en la Sociedad de la Información. 12(3), 56-75 [Fecha de consulta: $\mathrm{dd} / \mathrm{mm} / \mathrm{aaaa}]$.

http://campus.usal.es/ revistas_trabajo/index.php/revistatesi/article/view/8483/8576

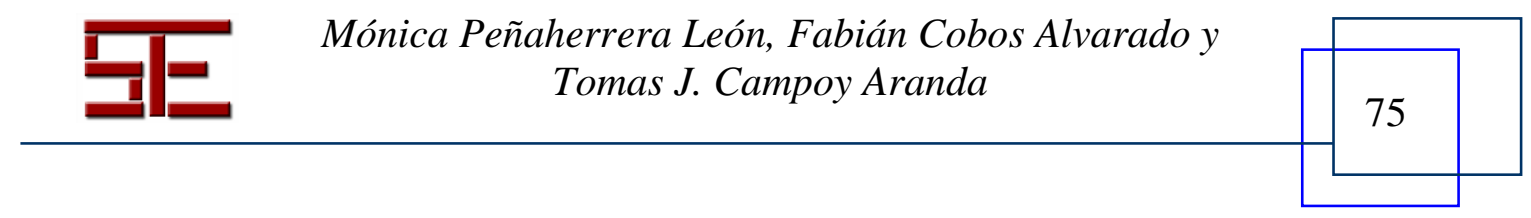

